
\title{
BOLETIN CRIMINOLÓGICO
}

Artículo 4/2012 (n. 136) Instituto andaluz interuniversitario de Criminología

\section{REALIDAD Y POLÍTICA PENITENCIARIAS}

Resumen: Tras demostrar la consolidada desvinculación entre los datos oficiales policiales y la realidad delictiva según las encuestas de victimización, el Observatorio de la Delincuencia (ODA) se ha planteado como un nuevo reto el análisis de la política penitenciaria y su contextualización en el marco de la actual política criminal. Así, en el nuevo informe se analiza en detalle la información de que disponen los autores en el ámbito penitenciario. El interés se centra fundamentalmente en conocer el impacto de una política penitenciaria que en los últimos tiempos parece haberse distanciado de los derroteros punitivistas en los que ha estado sumergida la política criminal en la primera década del siglo XXI. En este artículo se presenta un resumen de los resultados del informe.

Palabras clave: población penitenciaria, políticas criminal y penitenciaria, tratamiento penitenciario, conflictividad.

Title: Prison policy and prison reality Abstract: After proving the solid dissociation between official police statistics and criminal reality according to crime surveys, the Observatorio de la Delincuencia (ODA) has set as its new challenge to analyse prison policy and its contextualisation within current criminal policy. Thus, in this new report the authors analyse in detail data within the penitentiary field; it is particularly focused in learning the impact of a prison policy that in these past few years has apparently detached itself from the punitive approach adopted by criminal policy during the first decade of the 21st century. This article presents a summary of the results of the report.

Keywords: prison population, criminal policy, prison policy, penitentiary treatment, conflict

Recepción del original:

20 mayo 2012

Fecha de aceptación:

15 junio 2012

\section{Introducción}

Las consecuencias penitenciarias que se pueden derivar de tener unas prisiones superpobladas abarcan tanto el aspecto de tratamiento como el regimental. El tratamiento penitenciario se puede ver mermado en la medida en que los recursos no alcancen a todos, y desde un punto de vista regimental la superpoblación puede conllevar tensiones que deriven en una mayor conflictividad dentro de prisión.

Ante el aumento de la población presa, consecuencia ajena a la verdadera evolución de la delincuencia, la política penitenciaria se ve obligada a repensarse. Este nuevo planteamiento, como veremos, pasa por compaginar la necesaria creación de nuevas plazas con el diseño novedoso de centros de atención especializados dirigidos a la reinserción, como es el caso de los centros de inserción social (CIS), unidades de madres y unidades dependientes, entre otras.

\section{Metodología}

Para la obtención de los resultados se ha procedido al análisis descriptivocuantitativo (longitudinal y transversal) de datos oficiales penitenciarios relacionados con población, infraestructura, reinserción social (programas de tratamiento, educación y cultura) y seguridad interior (motines, suicidios, etc.). Los datos no publicados y necesarios se solicitaron a la Dirección General de Instituciones Penitenciarias. Para completar el panorama nacional se ha recurrido igualmente a los datos aportados tanto por el anuario estadístico de Cataluña como por las estadísticas básicas de ejecución penal elaboradas por los servicios penitenciarios catalanes.

Por lo que respecta a la comparación internacional, la información utilizada es una combinación de los datos aportados por el Council of Europe Annual Penal Statistics (SPACE I, 2009), los datos recogidos en el Prison Brief por el International Centre for Prison Studies (ICPS) y la información ofrecida por el Eurostat.

Por consiguiente, tanto en el análisis de los datos nacionales como en la comparación internacional, recogemos
UNIQ - ULIO 2012

Araceli-Aguilar Conde Elisa García España José Becerra Muñoz

información que hace referencia al total de la población penitenciaria española, lo que supone toda una novedad en el panorama científico actual que suele aludir exclusivamente al territorio competencia de la SGIP o a Cataluña.

Acabada la aproximación descriptiva-cuantitativa de los datos oficiales que habíamos recabado procedimos a realizar, como técnica complementaria, entrevistas telefónicas semi-abiertas a expertos en la materia. Para ello, hemos considerado a académicos especialistas en materia criminal y/o penitenciaria, a políticos de Instituciones Penitenciarias (IIPP) y a gestores del medio penitenciario. El objetivo era conseguir las diferentes valoraciones que pueden derivarse del análisis de los datos, así como explorar posibles hipótesis de trabajo en el ámbito penitenciario. La selección de los expertos se hizo a través de la técnica de la bola de nieve. Se realizaron un total de 18 entrevistas.

\section{Resultados}

A nivel internacional España se sitúa por encima de la media europea y con cifras superiores a todos los países de nuestro entorno europeo-occidental. Especialmente destaca España en las tasas de población femenina, ocupando la primera posición en Europa, y en las de población extranjera extracomunitaria, con un $12 \%$ por encima de la media europea.

De gran interés ha sido la información relativa a la cuantía de las condenas, el tiempo medio de estancia en prisión y el número de entradas durante el periodo objeto de estudio. A raíz de estos factores hemos comprobado que el alto nivel de población penitenciaria de nuestro país no responde a cifras altas de entradas a prisión. Así, la responsabilidad de la alta población penitenciaria española sólo puede descansar en el tiempo de estancia real de los internos en prisión. Este hecho se confirma al observar que España se encuentra en una posición muy alta en cuanto a estancia media en prisión por meses, sólo superada por Portugal y por el dato rumano que casi triplica al portugués. 
La población penitenciaria en España refleja un constante aumento. En la última década se ha pasado de 111 internos por cada 100.000 habitantes a casi 157 . En la primera década del presente siglo las prisiones españolas han tenido una media del $92 \%$ de hombres, frente al $7,94 \%$ de mujeres y una media del $77,5 \%$ de penados frente al $22,4 \%$ restante de preventivos. Además, la clasificación de penados en primer grado se encuentra en un constante descenso desde 2003. En el segundo grado se acumula, como es bien sabido, el grueso de penados (normalmente en torno al 80\% de los mismos). A lo largo de la década, en este grado se observan ligeras fluctuaciones de pequeño rango, alrededor del $5,4 \%$. Estas fluctuaciones podrían dividirse en tres periodos: el primero, del año 2000 al 2006, se caracteriza por un descenso constante y leve que va del $80 \%$ al 78\%; el segundo periodo abarca desde el año 2006 hasta el año 2009, produciéndose una estabilización en torno al 78\%; y finalmente, en el año 2010 se produce un repunte que lleva el segundo grado a valores en torno al $83 \%$. En tercer grado encontramos una evolución inversa a la del segundo grado, lo que sugiere que estos dos grados se retroalimentan. El delito más grave cometido por el $65 \%$ de los internos es, bien uno contra el patrimonio y el orden socioeconómico bien uno contra la salud pública.

La evolución del volumen de presos extranjeros presenta una tendencia ascendente en los últimos años en España, aunque en 2010 se aprecia un ligero receso, llegando al 35,6\% de la población presa. El número de preventivos extranjeros viene descendiendo desde 2003, con un $34,1 \%$, hasta el $30 \%$ alcanzado en 2010. Aunque se dé este descenso, hay que tener en cuenta que los preventivos extranjeros son notablemente más numerosos que los nacionales. Con toda probabilidad es la situación de desarraigo, y su consiguiente riesgo de fuga, la que hace que los jueces adopten con más frecuencia la medida cautelar de prisión preventiva con los extranjeros que con los españoles. Como consecuencia de ello, la situación procesal de los extranjeros en prisión puede ser una desventaja de cara a las posibilidades de disfrutar de recursos orientados a la reinserción social.

En cuanto al total de jóvenes menores de 21 años y de ambos sexos en las cárceles españolas los datos reflejan una ligera tendencia descendente a lo largo de la década.

Si analizamos la tasa de población penitenciaria en las diferentes regiones españolas las ciudades autónomas de Melilla y Ceuta aparecen a la cabeza de población penitenciaria en términos relativos, ya que ambas tienen tasas que se encuentran en torno al triple de la española. También Castilla y León supera los 200 presos por cada 100.000 habitantes, situándose en tercer lugar, para pos-

Gráfico $\mathrm{n}^{0}$ 1: Internos beneficiados por permisos de salida en la última década en España, centros de SGIP y Cataluña (\%)

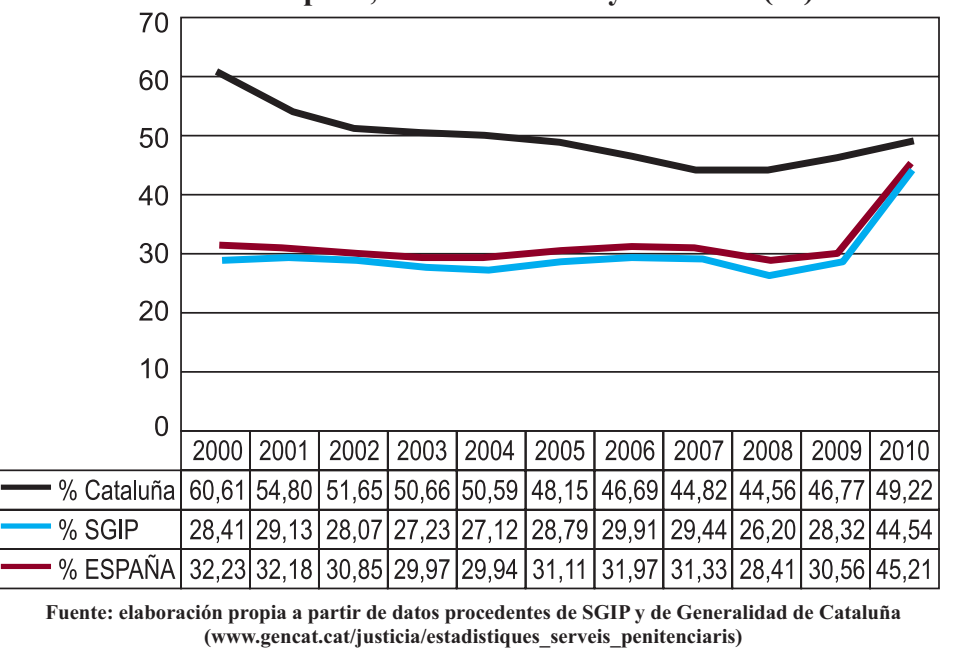

teriormente dar paso al grueso de las comunidades, que se sitúan en la franja de 100 a 200 presos por cada 100.000 habitantes. Las tres comunidades con una tasa de menos de 100 internos son Murcia, País Vasco y Navarra.

La alta tasa de población penitenciaria en España está acompañada, además, de uno de los índices de ocupación más altos de toda Europa, que refleja un sistema saturado, al 141\% de ocupación, 37 puntos por encima de la mediana europea. En el ámbito nacional, en 2010 todas las comunidades tienen una media de ocupación por encima del $100 \%$. De entre ellas, Melilla aparece con la mayor tasa $(269,44 \%)$, más de 40 puntos por delante del País Vasco (231,48\%), la segunda comunidad con mayor masificación penitenciaria. A partir de ahí, los saltos descendentes son mucho menos abruptos. Cataluña es la comunidad autónoma con menos masificación, con un $94,3 \%$ de ocupación para el año 2009.

Respecto a la política penitenciaria, existen estrategias que se centran bien en mejorar el clima de convivencia y de respeto entre los internos (módulos de respeto), bien en atender las necesidades específicas de un determinado colectivo, bien en aumentar los contactos del interno con el exterior, disminuyendo el tiempo de reclusión del interno (módulos de respeto, terceros grados, medios telemáticos, libertad condicional, etc.)

El establecimiento de módulos de respeto en las prisiones dirigidas por IIPP es progresivo desde 2007 y el número de internos participantes en ellos en el territorio SGIP es siempre ascendente desde esa fecha $(5,04 \%)$ a $2010(17,66 \%)$.

Un elemento definidor de la política penitenciaria lo constituye, en nuestra opinión, el número de traslados y los permisos de salida, ya que a través de ellos se puede deducir la respuesta dada a las diferentes necesidades de los internos y el aumento del contacto del interno con el exterior al disminuir el tiempo de reclusión en los centros. En relación a los traslados, las causas más frecuentes para que se concedan son: razones médicas, asunto extraordinario y vinculación familiar. En cuanto a los permisos de salida, en España se observa que desde el año 2000 hasta el 2008 el volumen de presos que se beneficiaban de los mismos se mantuvo en torno al $30 \%$, produciéndose un aumento en el número de concesiones en el año 2009. Es lo que se observa en el gráfico ${ }^{\circ} 1$, donde se aprecia también la evolución experimentada en las prisiones de SGIP y de Cataluña.

La concesión de terceros grados también nos permite pulsar la repercusión de la política penitenciaria, al producirse a través de ellos la excarcelación de aquellos sujetos que cumplen con determinadas características. Ciertos factores relacionados con el tercer grado han sufrido modificaciones, especialmente en el territorio de la SGIP, con la implantación y progresiva generalización de los CIS, a través de los que se pretende impulsar la inserción social de los internos en las últimas etapas de su condena. Desde el año 1993 hasta 2010 se han construido 29 CIS, de los cuales 27 fueron creados a partir de 2004, siendo en 2009 cuando más centros se abrieron.

Otra modificación relativa al tercer grado es la implantación progresiva de mecanismos de control que descansan en las nuevas tecnologías, concretamente el uso de medios telemáticos. Estos sistemas permiten conceder un mayor margen de confianza al sujeto y adelantan la independencia de los internos. Su implantación se muestra en el gráfico $n^{\circ} 2$.

El gráfico $\mathrm{n}^{\circ} 3$ muestra cómo ha sido la evolución de la concesión de libertades condicionales en España. Ésta ha sufrido un fuerte descenso desde el año 2000 hasta el 2004, a partir del cual los valores se mantienen estables en torno al 9\% hasta el año 2008, en el que se experimenta un ascenso marcado y progresivo que se mantiene hasta el final de la década.

La política penitenciaria también puede ser evaluada mediante el análisis de los programas de tratamiento peniten- 


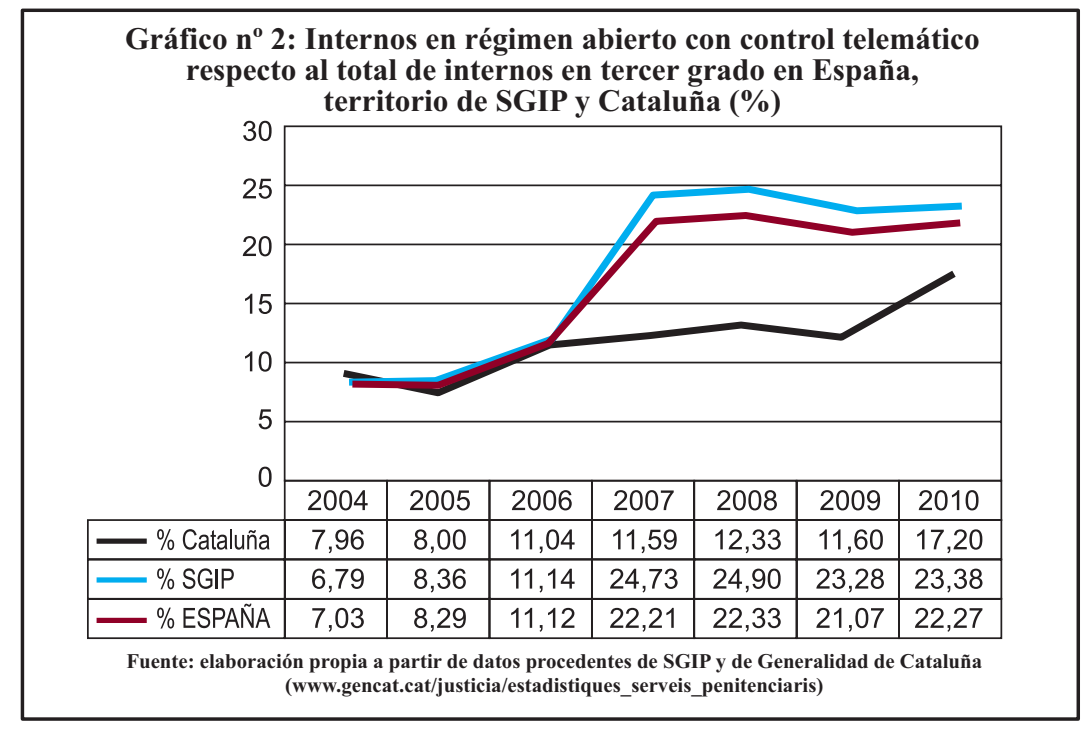

Gráfico $\mathrm{n}^{0}$ 3: Internos a los que se les concede la libertad condicional en la última década respecto al total de población penitenciaria en España, territorio SGIP y Cataluña (\%)

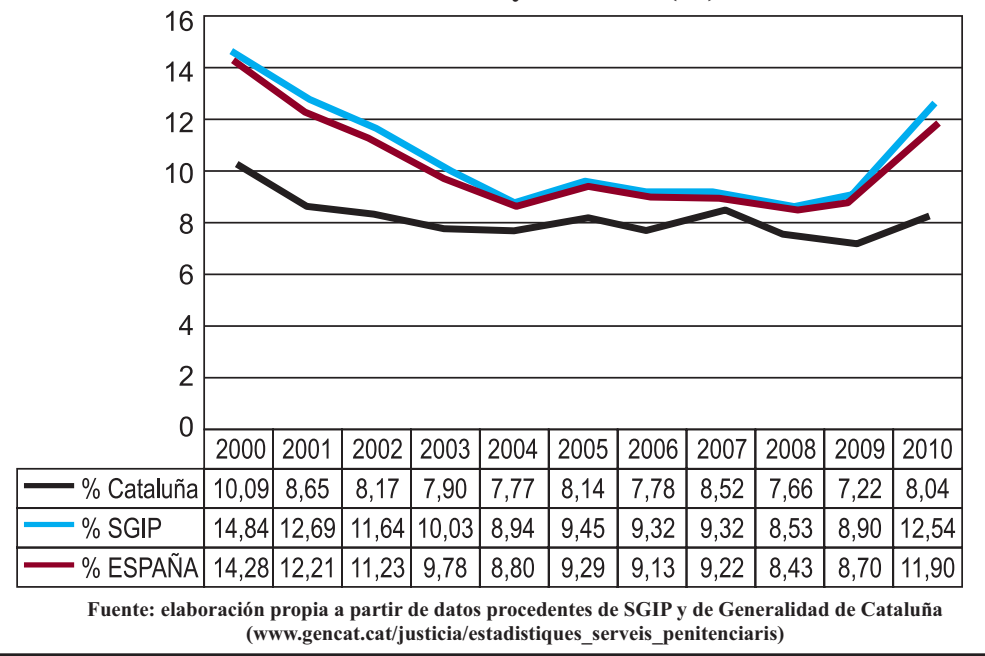

ciario. Su contenido y extensión nos puede dar información relevante sobre la evolución de la política penitenciaria española, así como valorar ésta en el contexto de la actual política criminal.

Entendemos por programas comunes aquellos que se están llevando a cabo de una forma similar tanto en territorio SGIP como en Cataluña. Todos muestran una evolución ascendente para el global de España. Entre ellos destacan: programas de ocio y cultura, deportivos, de intervención con agresores sexuales, de intervención para agresores de violencia de género, de prevención de suicidios, de intervención con drogodependientes, etc.

Se han denominado programas comunes no agrupables a aquellos que tienen la misma naturaleza y están orientados hacia el mismo fin pero que se aplican de manera diferente en los centros catalanes y en los del territorio de la SGIP. Entre éstos están el programa de intervención con jóvenes y el programa de intervención con personas extranjeras.

En relación al primero, aunque este tipo de programa se ofrece en toda España no se implementa del mismo modo en
Cataluña que en el resto del país. En todo caso, en los últimos años este programa se aplica cada vez a más jóvenes en los centros penitenciarios de la SGIP (para los centros catalanes no hemos podido contar con información). En 2007 el $4,69 \%$ del total de reclusos jóvenes estaba en este programa de tratamiento, mientras que en 2010 el porcentaje subió hasta $19,02 \%$.

El segundo programa es llevado a cabo por todos los centros penitenciarios de territorio nacional, pero no ha sido posible sumar los datos de Cataluña a los del resto de España ya que la forma de implantación en ambos territorios es diferente. El programa adopta un enfoque integral de intervención a través de programas generales (para aquellos que conozcan el idioma) y de programas específicos (para los que no lo conozcan). En este programa la intervención penitenciaria se ve limitada a consecuencia de las medidas de excarcelación previstas para extranjeros. Desde el año 2007 el porcentaje de presos extranjeros inscritos en el programa marco de intervención va desde el 1,05\% en 2007 hasta el 4,06\% en 2010 .
Además de los dos conjuntos de programas anteriores, existen otros que se están aplicando en territorio de la SGIP $\mathrm{y}$ no nos consta que se apliquen en Cataluña, y viceversa. Por tanto, nos queda la duda de si los programas sobre los que no hemos encontrado suficiente información se están llevando a cabo en ambos territorios. Son los que hemos llamado programas no comunes.

Así, nos consta que en Cataluña se está aplicando un tratamiento de conductas delictivas violentas, que experimenta una ligera evolución ascendente desde el 2008 (con un 3\% de reclusos catalanes insertos en este programa), hasta el 2010 (con un 4\%).

A su vez, en las prisiones de la SGIP se tiene constancia de un programa de terapia asistida con animales (TACA), que resulta útil para internos con problemas de afectividad o autoestima. El porcentaje de internos adscritos a este programa es escaso aunque con una evolución ascendente desde el 2007 $(0,11 \%)$ hasta el $2010(0,41 \%)$.

Además de todo lo comentado hasta el momento, se debe tener en cuenta que la convivencia obligada en un lugar cerrado, reducido, hacinado y falto de intimidad puede generar violencia y tensión entre las personas presas, así como entre éstas y el personal que trabaja en prisiones. De ahí que sea lógico pensar que un aumento de población penitenciaria conlleve un aumento de la conflictividad y por tanto de los incidentes regimentales.

Sin embargo, los fallecimientos $y$ suicidios en prisión han tenido una tendencia descendente en los últimos años. Por su parte, el número de faltas cometidas por los internos entre 2007 y 2010 ha aumentado en términos absolutos de 50.182 a 60.897 en las prisiones españolas. Pero teniendo en cuenta el aumento de población penitenciaria, la tendencia, en términos relativos, permanece estable en el periodo de tiempo estudiado. La misma tendencia se observa en las agresiones a funcionarios a lo largo de los últimos cinco años. Por el contrario, las agresiones a internos presentan un signo ascendente, llegando casi a duplicar en términos relativos la cantidad de agresiones de 2010 (6\%) a las de $2006(3,7 \%)$. No obstante, el $90 \%$ de las agresiones, tanto a funcionarios como a internos, producidas en las prisiones del país han sido calificadas como leves.

Con todo, el perfil que presenta la evolución de las agresiones a funcionarios en toda España no se reproduce en Cataluña. En esta comunidad autónoma la tendencia es ascendente y muy pronunciada entre $2006(0,3 \%)$ y $2009(1,3 \%)$, distanciándose de la del territorio SGIP, que se mantiene estable el resto del quinquenio. En el caso de las agresiones a internos, se aprecia, dentro de la tendencia ascendente general, un mayor volumen en Cataluña que en el resto del país. 
Las evasiones que se producen son excepcionales. En términos absolutos, los datos en España oscilan entre 56 y 83 evasiones en el quinquenio comprendido entre 2006 y 2010. En cuanto a la inutilización grave de dependencias, plantes y motines, según los datos de la SGIP (no disponemos de datos catalanes) en 2006 se produjeron 5 motines y 2 en 2007; desde entonces no se han vuelto a producir sucesos de este tipo. Los plantes y las inutilizaciones graves de dependencias son más numerosos, siendo su tendencia descendente desde 2007 a 2010.

\section{Una valoración global de los datos por expertos}

Los datos recopilados se presentaron a expertos académicos, políticos y gestores del ámbito penitenciario en entrevistas semi-estructuradas y telefónicas. A continuación se exponen de forma muy resumida algunos de los temas tratados con ellos:

1.- La mayoría de los entrevistados apunta, como motivo principal de la alta tasa de encarcelamiento en nuestro país, a las características de nuestro sistema penal, que hacen que sea fácil entrar en prisión y difícil salir de ella, especialmente para los inmigrantes. Según los expertos, entre los mecanismos que facilitan la entrada en prisión destacan el uso excesivo de la prisión preventiva y el escaso uso de penas alternativas en general. Se denuncia la utilización desproporcionada de la prisión provisional con la población inmigrada, por falta de arraigo social y por el temor a que se evadan de la acción de la justicia. Entre las dificultades para salir de la prisión se apunta a la extensión de las penas de prisión y a los obstáculos para acceder a mecanismos de reducción de la condena, dada la desaparición de la redención de penas por el trabajo, el establecimiento de periodos de seguridad que dificultan el acceso al tercer grado o el endurecimiento del acceso a beneficios penitenciarios. Se alude al excesivo acercamiento de la pena nominal a la real.

2.- Existe una opinión prácticamente unánime de que, si bien la tasa de ocupación de las prisiones podría conllevar un mayor número de conflictos, lo cierto es que tal afirmación no parece verificarse en la realidad penitenciaria española. Al respecto se dan diferentes explicaciones: algunos señalan la facilidad para ocultar conflictos dentro de prisión, aunque los funcionarios de prisiones con tareas de vigilancia entrevistados aseguran no haber notado un incremento de conflictos a pesar del crecimiento de la población penitenciaria; otros apuntan al cambio en el perfil de los presos en los últimos veinte años, en la medida en que actualmente los niveles de violencia, marginación y drogadicción son menores; un argumento parcialmente contrario se sitúa en la existencia de drogas y su papel calmante para los internos, evitándose así estallidos de violencia; la gestión de los beneficios penitenciarios en función de la buena conducta del interno también contribuye al mantenimiento de la disciplina carcelaria; por último, el diseño arquitectónico de las prisiones aumenta la dificultad para organizar motines o fugas. Además, se añade, la vida en prisión asegura un nivel de bienestar alto aludiendo al "estilo mediterráneo" que impera en las prisiones españolas, donde se permite la movilidad de los presos entre los distintos espacios de la prisión. Una opinión particular considera que la ausencia de estallidos de motines no es sintomático de la verdadera tensión que se vive en las cárceles españolas, argumentando que la ausencia de motines se debe a la "falta de estructuras de oportunidad" tanto en el interior como en el exterior que sirva de sostén a las expresiones violentas de los internos.

3.- Los datos ponen de manifiesto, tal como ratifican los expertos, que la política penitenciaria de la última legislatura del PSOE podría entrar en contradicción con las características de una política criminal que viene siendo en los últimos años cada vez más rigurosa El aumento en la concesión de permisos de salida, especialmente en 2010 en el territorio de la SGIP, o el gran uso de los medios telemáticos en terceros grados que se está produciendo en toda España permiten apuntar una tendencia hacia la excarcelación, diferente a la de épocas anteriores. Algunos entrevistados aluden a que la sociedad es destinataria de un discurso securitario que prioriza la dureza contra el crimen, contundencia que luego se ve amortiguada por un sistema penitenciario que se orienta en sentido opuesto. Hay quien justifica las diferencias existentes entre ambas políticas en los distintos principios informadores, ya que en la penitenciaria priman la reinserción y el tratamiento. Para algún experto el sistema de ejecución modula, atenúa y rectifica los objetivos del sistema penal. Tendría sentido, por tanto, la tesis de que el sistema penitenciario atiende a sus propias necesidades, configurándose como un ámbito pragmático que pretende solucionar problemas concretos, y necesitado de ciertas válvulas de escape frente a la saturación del sistema. Esto trae como consecuencia excarcelaciones que tienen más que ver con la alta tasa de ocupación que con la auténtica rehabilitación de los internos.

4.- Se preguntó a los expertos si consideran que se ha producido un cambio en la política penitenciaria española. Casi todos los académicos, políticos y gestores reconocen este cambio. Desde IIPP se estima que los cambios son una nueva fase de la "evolución hacia la humanización" con la que se encuentra comprometida la política penitenciaria española. La mayoría coloca el punto de inflexión en la llegada de la Directora General, Mercedes Gallizo, en 2004, y sus actuaciones encaminadas a adelantar las excarcelaciones a través de los terceros grados. Algunos piensan que hay una clara tendencia de nueva política penitenciaria basada en la potenciación del régimen abierto y en acciones que buscan mejorar la convivencia. Se refieren a la construcción de los CIS como un aspecto que concreta una política especialmente sensible y diferente a la de legislaturas anteriores, más basadas en "el orden, la seguridad y el control". Se dan interpretaciones antagónicas sobre los motivos que han llevado a ese cambio en la política penitenciaria. Si bien todos coinciden en que hay un deseo de descongestionar las prisiones, hay un conjunto de posturas más amables con respecto a las intenciones de esa política, al considerarla desinteresada y basada en una cuestión de humanidad y justicia social. Otros apuntan que la nueva política penitenciaria sólo pretende dar respuesta a las necesidades que España tiene de proyectar una imagen diferente frente a Europa y de reducir el gasto que suponen los actuales niveles de población encarcelada, llegando a afirmar que la masificación ha sido la que ha impuesto las novedades. La primera postura intuye que la actual política está siendo efectiva tanto desde el punto de vista de la descongestión como del de la reinserción, mientras que la segunda considera que se trata simplemente de una cuestión de imagen, sin que ello tenga efecto alguno en la reinserción de los presos.

5.- No existe unanimidad a la hora de determinar cuáles deberían ser las prioridades en el ámbito penitenciario. Las propuestas de los expertos son tan diversas como entrevistas hemos realizado. Alguna de esas propuestas apunta a la necesidad de abordar la cuestión penitenciaria anteponiendo los derechos de las personas sobre la eficacia de la gestión. Otros establecen como prioridad el análisis científico de la realidad penitenciaria, lo que contribuiría tanto a un mejor conocimiento de las condiciones de vida de los presos como a una mayor transparencia en la gestión. El resto de propuestas han sido algo más concretas y se han centrado en diversos aspectos como: condiciones laborales, estructura carcelaria, colectivos específicos, prioridades en régimen penitenciario, prioridades de tratamiento o asuntos procesales. Todos los expertos prefieren la reforma del Código penal como el medio más adecuado para alcanzar la coordinación entre las políticas penitenciaria y criminal, así como para el objetivo común de descongestión carcelaria. Se alude de forma reiterada y se ve como una necesidad imperiosa que la propuesta reforma del Código penal atienda a dos finalidades: profundizar en las penas alternativas a la prisión y acortar las penas abstractas.

Puede encontrar información sobre los autores y el Observatorio de la Delincuencia en:

www.oda.uma.es 